\section{Student reflection}

PALABRAS CLAVE • SOSTENIBILIDAD · PATRIMONIO MEDIOAMBIENTE · MEMORIA · CULTURA

KEYWORDS • SUSTAINABUUTY · HERITACE · ENYIRONMENT . MEMORY · CULTURE
RESUMEN

En esta oportunidad presentamos los resultados de dos trabajos realizados por estudiantes de pregrado de la Facultad de Arquitectura y Urbanismo de la Universidad de Chile. Por un lado, la investigación Cálculo de vida útil estimada para el reciclaje sostenible de industrias abandonadas de Daniela Carter y por otro lado, el proyecto El cementerio flotante de Fabián Leiva.

\section{ABSTRACT}

Herewith we present the results of two works carried out by undergraduate students of the Faculty of Architecture and Urbanism of Universidad de Chile; one titled Estimated lifespan calculation for the sustainable recycling of abandoned industries of Daniela Carter, and the other one, Floating cemetery of Fabian Leiva.

\title{
Reflexión estudiantil
}

\section{INTRODUCCIÓN}

Desde perspectivas distintas, los dos trabajos que aquí se presentan nos recuerdan que la arquitectura es una manifestación cultural, íntimamente vinculada con las problemáticas que determinan y condicionan el desarrollo de nuestras sociedades. En el caso de la investigación "Cálculo de vida útil estimada para el reciclaje sostenible de industrias abandonadas", Daniela Carter centra su atención en el ciclo de vida de los edificios, un asunto fundamental para pensar rehabilitaciones sostenibles sobre obras en desuso o que reclaman una renovación programática para atender a nuevos requerimientos. Por su parte, el proyecto de título de Fabián Leiva, "El cementerio flotante", plantea una aproximación sensible a la comunidad Pehuenche, a sus tradiciones y costumbres, a su forma de entender el territorio, continuamente olvidadas por la lógica del progreso. Así, ambas miradas sugieren la necesidad de desarrollar una arquitectura debidamente situada, atenta a los requerimientos medioambientales y culturales de aquella comunidad que la habita, usa y modifica.

\section{CÁLCULO DE VIDA ÚTIL ESTIMADA PARA EL RECICLAJE SOSTENIBLE DE INDUSTRIAS ABANDONADAS*}

LIC. DANIELA IVONNE CARTER · Santiago, Chile · dcarter@ug.uchile.cl Fecha de recepción: 28 de septiembre de 2018 - Fecha de aceptación: 28 de noviembre de 2018

\section{RECICLAJE SOSTENIBLE}

Reciclar una infraestructura obsoleta, en desuso y abandono, involucra acondicionarla o intervenirla, es decir, "someter el material usado a un proceso para que se pueda volver a utilizar" (RAE, 2016). Desde esta perspectiva, se infiere que los materiales y edificios, tienen la capacidad inherente de prolongar el ciclo de vida útil, interviniéndolos de acuerdo a la condición de adaptabilidad y compatibilidad de sus elementos con el nuevo uso, realizando una "re-programación funcional" o proceso de re-codificación, mediante una nueva lectura espacial (Lillo, 2010).

\section{VIDA ÚTIL DE LAS CONSTRUCCIONES (ISO 15686)}

Se tiende a confundir los conceptos de vida útil y durabilidad. La vida útil de un edificio se define como el período de tiempo desde la construcción hasta la pérdida de utilidad de alguna de las partes o de la totalidad del edificio según el uso original, vale decir, corresponde al período de tiempo en que funciona correctamente (ISO, 2017). La durabilidad, en cambio, es un factor que incide en la vida útil y se entiende como la capacidad para desempeñar la función o programa original del edificio sin sufrir alteraciones físico-químicas en

* Realizado en base a los resultados del seminario de investigación "Reciclaje sostenible de industrias abandonadas y compatibilidad de oficinas según criterios y normas internacionales de eficiencia energética", desarrollado por la autora durante el semestre de otoño de 2017 en la Facultad de Arquitectura y Urbanismo de la Universidad de Chile (Profesora guía: Jeannette Roldán Rojas). 
A1. Vida Útil de Diseño por categoría y usos de edificios. Fuente: Elaboración propia, según la Norma ISO 15686.

un determinado período de tiempo, es decir, es la capacidad que tiene el edificio para funcionar en óptimas condiciones, sin deteriorarse (CSA, 2001).

Estimar la vida útil y durabilidad del edificio, al igual que la de los materiales y partes que lo componen, permite realizar el diseño del proyecto de reutilización o reciclaje con proyecciones de sostenibilidad en el tiempo, prever impactos ambientales y costos de mantención por sustitución de alguna de las partes que no funcionen en óptimas condiciones, con el fin de ejecutar las modificaciones necesarias para evitar futuras reparaciones (BRE, 2007) (Ortega, L. et al., 2015).

A partir de la norma ISO 15686, considerando el Diseño por Ciclo de Vida (DCV) se infiere que la vida útil comprende las etapas de la construcción y del uso/operación/mantenimiento del edificio hasta que este deja de funcionar, tras el abandono o la demolición, ya que los conceptos guardan estrecha relación con el funcionamiento óptimo del edificio (Hernández, S., 2011$)$ (ISO, 2017).

La estimación del cálculo de VUE, en la etapa de diseño e intervención de los edificios preexistentes, debe tener en cuenta el rendimiento de la construcción o capacidad física de cumplir la función para la cual el inmueble fue destinado, todo esto con el objetivo de tomar decisiones rentables a largo plazo (Hernández, S., 201 1), referente a:

- Sistemas constructivos.

- Costos ligados a restauraciones, reparaciones y reemplazo de elementos.

- Tipos de materiales, tecnologías de construcción y mano de obra.

- Plan de gestión de mantención del edificio.

- Costos por ciclo de vida del edificio e impactos ambientales implicados.

De acuerdo con la Norma ISO 15686 : "Buildings and constructed assets - Service life planning", se plantean cuatro tipologías de vida útil:

\section{(1)}

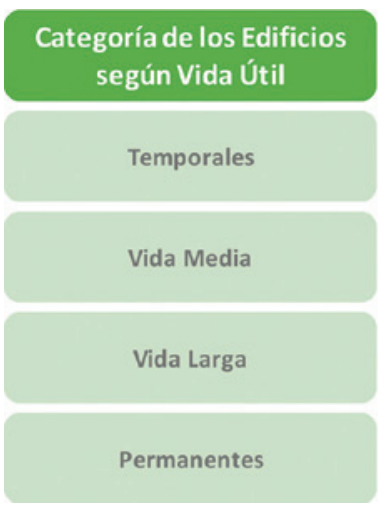

\begin{tabular}{|c|}
\hline $\begin{array}{c}\text { VUD Vida Útil de Diseño } \\
\text { por Categoría (años) }\end{array}$ \\
\hline 0 - 10 Años \\
\hline 25 - 49 Años \\
50 - 99 Años \\
Más de 100 Años
\end{tabular}

Descripción de Tipologías de los Edificios

Construcciones no permanentes, es decir, son
provisionales
Faenas / Ferias / Exposiciones
Edificios Industriales / Estacionamientos
Edificios Residenciales
Comercio / Oficinas
Salud / Educación

Edificios Patrimoniales/ Monumentales Edificios Públicos: Museos, Galerías y Bibliotecas
- VUD (Vida Útil de Diseño): Estimada por el proyectista según el uso del edificio.

- VUE (Vida Útil Estimada): Estimada según materiales, características del proyecto, relación de la construcción con el entorno en términos de adaptación a variables que inciden en el deterioro de los materiales e infraestructura, técnicas y gestión de uso y mantención.

- VUREF (Vida Útil de Referencia):

Proyectada para un edificio y sus partes en un determinado conjunto con ciertas condiciones de uso.

- VUR (Vida Útil Residual): Período de vida útil entre desuso y reutilización. También se le define al período de vida útil restante de un elemento en determinado momento hasta que se deteriora.

La VUD se obtiene de acuerdo con la tipología de uso del edificio según la norma ISO 15686, tal como se indica a continuación:

De la Figura A1 se desprende que las industrias poseen una VUD de 25 a 49 años y que, al ser destinadas a nuevos usos, según las categorías de vida larga o permanente se incrementa, en función de la nueva tipología que contemple: edificios residenciales, comercio, oficinas, salud, educación o edificios públicos y patrimoniales, en un margen de $25-50$ y 59 60 años, respectivamente. Se infiere, en ello, cierta presunción de la Norma Internacional
ISO 15686 respecto al grado de preservación de estos últimos, considerando que, en dominio del Estado, permanecerán operativos y abiertos al público y en perfectas condiciones, debido a una adecuada gestión de mantención postocupacional. En definitiva, tanto los edificios públicos y patrimoniales estarán a cargo de instituciones responsables de conservarlos sin daños y deterioro.

Según la norma ISO 15686, es posible evaluar la VUE de las edificaciones, a partir del producto de 7 factores de durabilidad del proyecto, identificados desde la $A$ hasta la $G$, indicados a continuación:

- $\mathrm{A}=$ Calidad del diseño arquitectónico y constructivo: Calidad de los trabajos a nivel de proyecto, en función del tipo de obra, la experiencia en el rubro y la certificación de los miembros o entidades encargadas (arquitectos, ingenieros, constructores y especialistas).

- $\quad \mathrm{B}=$ Calidad de los materiales de la construcción: Calidad de los materiales en función del rendimiento, los procesos de fabricación y el cumplimiento de las necesidades funcionales en relación con las normas técnicas, estándares ambientales y certificaciones.

- $\quad$ C = Calidad de acondicionamiento interior del edificio: Habitabilidad interior del proyecto en términos de la temperatura, la 
ventilación, la iluminación y la humedad interior, estos influyen en el deterioro de los componentes. Dependerá directamente del diseño bioclimático.

- $\quad \mathrm{D}=$ Influencia del entorno del edificio: Grado de exposición a las variables climáticas exteriores, tales como viento, humedad, lluvia ácida, contaminación y radiación solar que afectan el deterioro de los componentes.

- $\quad \mathrm{E}=$ Calidad de la mano de obra: Calidad de los trabajadores de la construcción en relación a las certificaciones, niveles de capacitación de los obreros y grado de especialización de la mano de obra.

- $\quad F=$ Uso del edificio y operabilidad del inmueble: Operabilidad y grado de uso del edificio previsto en función del programa y destino que influye en la degradación y deterioro de los componentes.

- $G=$ Grado de mantención: Nivel de gestión de la preservación del edificio en período de postocupación, considerando la frecuencia de procedimientos y el nivel de especialidad de los encargados.

Cada factor, de acuerdo con las descripciones señaladas, permite realizar el análisis de un edificio a la hora de intervenirlo, aplicando criterios de valorización de estos, según índices alto $(1,2)$, medio $(1,0)$ y bajo $(0,8)$ aplicándolo a la fórmula:

- $\mathrm{VUE}=\mathrm{VUD} \times \mathrm{A} \times \mathrm{B} \times \mathrm{C} \times \mathrm{D} \times \mathrm{E} \times \mathrm{F} \times \mathrm{G}$, que simplificándose queda expresada de la siguiente manera: VUE $=$ VUD $\times 1,2^{n} \times 1,0^{n}$ $x 0,8^{n}$, donde $n$ corresponde a las veces en que se repite la valorización para cada uno de estos factores.

Esta metodología es empleada cuando el acceso a los datos, referente al rendimiento de los materiales y elementos constructivos de una obra es limitado, evaluando estos factores con una lógica integral y considerando la complejidad del sistema (Marteinsson, 2005). Al analizar la VUE de los edificios en etapas previas a las intervenciones, permite contar con una información útil de los requerimientos del proyecto con el fin de mejorarlo, generando un diseño sustentable, que considere diversos aspectos, tales como el rendimiento y durabilidad de los sistemas y subsistemas que contempla el edificio original, costos por ciclo de vida del edificio, con las respectivas gestiones de mantención en la etapa de postocupación, teniendo en cuenta además la expectativa de vida del diseño y los materiales (BRE, 2007) (Hernández, S., 2011 ) (Hernández, S. et al., 2015).

La VUREF implica la evaluación de cada uno de los elementos respecto al conjunto, considerándose adecuado solo para efectuar análisis previo, cuando se trata de remodelaciones y restauraciones, pues está ligado al deterioro y a la vida útil de los materiales y no al uso del inmueble, ya que no se considera en el análisis del reciclaje del caso estudio.

Finalmente, la VUR consiste en el cálculo simplificado del período de años transcurridos desde que el edificio cesa las funciones hasta que se inicia la construcción del proyecto de reutilización o reciclaje, por lo que basta con efectuar una resta entre el año reciclaje menos el año cierre del destino original. Este dato no aporta mayor análisis, solo informa el tiempo en el que un edificio ha estado en desuso.

Sin embargo, de las tipologías de vida útil mencionadas, solo dos son apropiadas para evaluar y tomar decisiones de reciclaje, la Vida Útil de Diseño (VUD) y la Vida Útil Estimada (VUE), la primera aplicada para el cálculo de la segunda, mediante el método de factores, utilizado para el análisis de un caso estudio en la presente investigación.

\section{METODOLOGÍA: CÁLCULO DE VUE POR PRODUCTO DE FACTORES (SEGÚN ISO 15686)}

El cálculo de vida útil estimada permitirá establecer un marco de comparación de calidad sostenible del proyecto de reciclaje en relación con el proyecto original, en función a las determinantes de durabilidad y deterioro.

De esto se infiere que un edificio que cumpla con una alta calidad del diseño y acondicionamiento interior, sumado a una óptima gestión de los procesos de construcción y una selección adecuada de los materiales, con sus respectivas certificaciones podrían tener una vida útil más prolongada.

En esta investigación se puso especial acento en el cálculo de vida útil estimada VUE, según Método de Cálculo de Factores de la Norma ISO 15686, con el fin de comprobar que el reciclaje de inmuebles aumenta la vida útil de un edificio en desuso, para esto se compararon los resultados obtenidos mediante la sistematización de fichas de evaluación.

Ambas fichas son similares y han sido elaboradas para esta investigación con el fin de generar un instrumento de cálculo simple y fácil de completar, pudiendo ser utilizado en futuras investigaciones e intervenciones de los edificios, teniendo en cuenta las descripciones de los factores de durabilidad antes mencionados y variando la VUD para otra tipología de usos, indicados en la FIgURA A1.

Teniendo en consideración el método de cálculo de vida útil estimada que estipula la norma ISO 15686, al establecer los mismos factores y comparar los rangos de períodos mínimos y máximos posibles de obtener, al reciclar un edificio, vale decir, reutilizarlo otorgándole un nuevo uso, para el caso de industrias a oficinas, es posible aumentar al doble la vida útil del edificio, tomando en cuenta que la vida útil de diseño (VUD), definida por tipología de uso es de por sí el 200\% (industrias 25-49 años, oficinas 50-99 años) (FIgURAs A2 y A3).

Así, considerando los niveles bajos $(0,8)$ en todos los factores de cálculo de la ficha, para las industrias el rango mínimo serán 5,24 a 89,57 años y el rango máximo de 10,27 a 175,57 años y para oficinas el mínimo de 10,48 a 179,15 años y el máximo de 20,76 a 354,73 años. 
A2. Ficha A: Cálculo de VUE por Método de Factores; Uso Original: Industrias. Fuente: Elaboración propia, a partir de la sistematización de la Norma ISO 15686. [ n: Número de repeticiones del factor. X1 y X2: años que definen rangos límites de la vida útil ].

A3. Ficha B: Cálculo de VUE por Método de Factores; Nuevo Uso: Oficinas y Comercio. Fuente: Elaboración propia, a partir de la sistematización de la Norma ISO 15686. [ n: Número de repeticiones de los factores alto, medio y bajo. X1 y X2: años que definen rangos límites de la vida útil ].

A4. Comparación entre vida útil estimada de industrias y oficinas, según factores mínimos y máximos. Fuente: Elaboración propia, a partir del análisis de la metodología de cálculo de VUE.

(12)
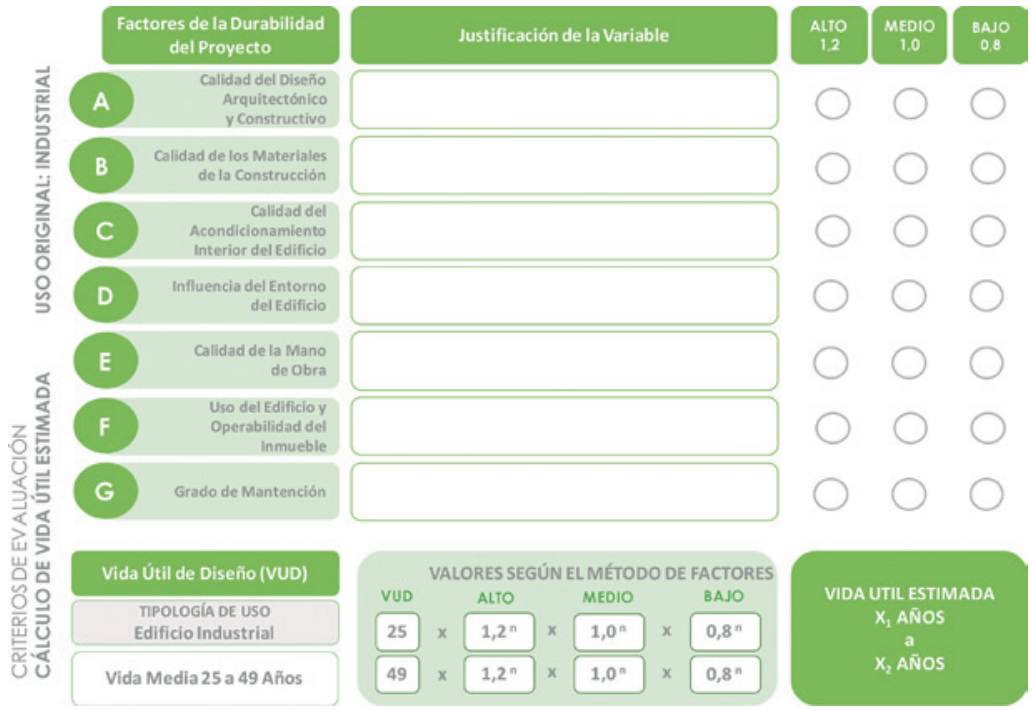

(4)

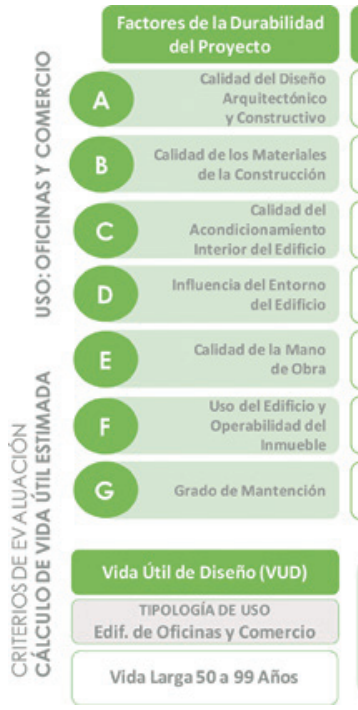

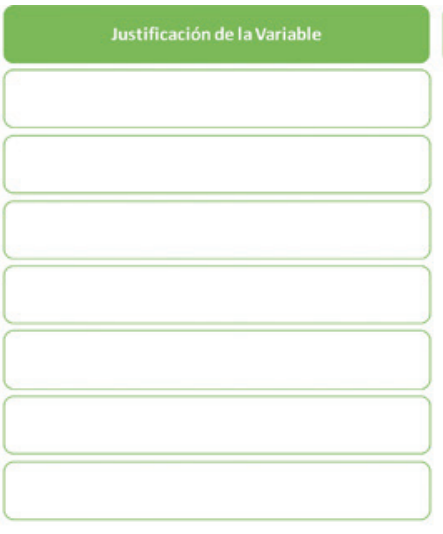
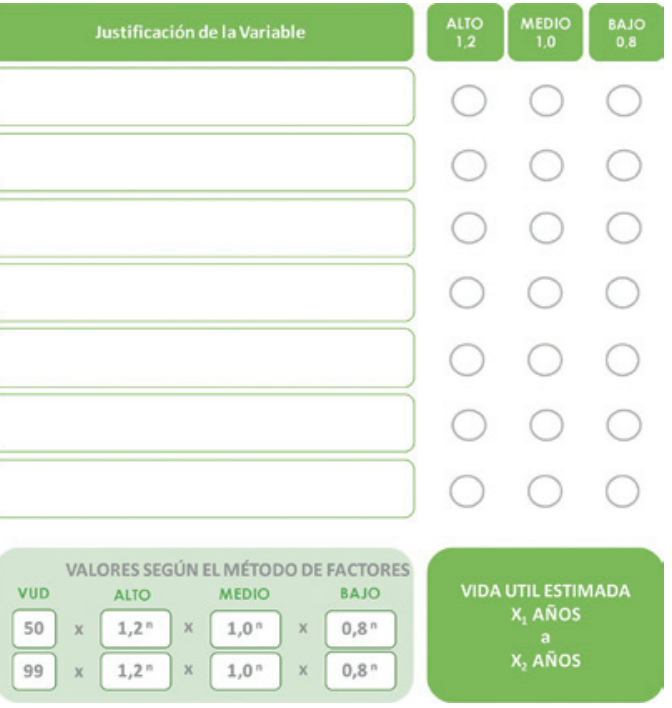

De lo anterior podemos inferir que el uso más conveniente para un edificio industrial, en términos de vida útil es un edificio de vida permanente (+ 100 años), los que serían de carácter patrimonial, monumental y/o público (FIGURA A4).

\section{CASO DE ESTUDIO}

CHV Machasa de Elton Léniz Arquitectos, es el reciclaje del edificio que correspondió a la exindustria textil Yarur S.A., que se ubica en la zona deteriorada del Anillo de Hierro de Santiago, en el pericentro urbano suroriente y se mantuvo en desuso durante 29 años (vida útil residual), tras el cierre de funciones en el año 1982.

El reciclaje es relativamente reciente y abarcó una superficie superior a $55.000 \mathrm{~m}^{2}$. El edificio principal tenía grandes dimensiones y una altura de 7,75 [m] que permitió contener gran cantidad de oficinas y estudios de televisión, dividiéndose en tres niveles; el subsuelo fue destinado a estacionamientos. Para su reutilización, solo se demolió el 8,93\% de la estructura de pilares estructurales de HA de la placa industrial, para
(44)

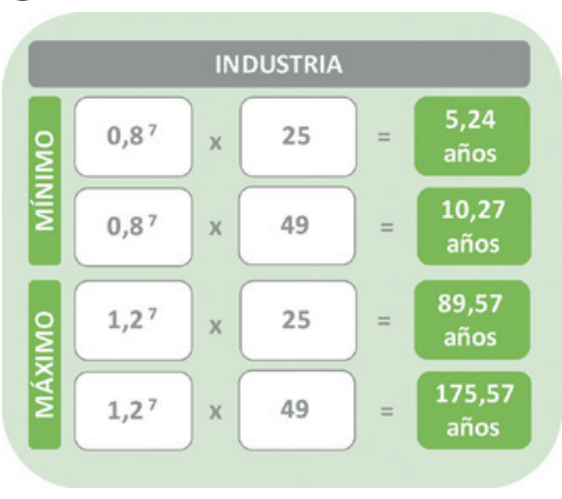

situar los sets de producción y solo el 0,5\% del pavimento para articular el acceso a los estacionamientos (FIGURA A5).

En la intervención se emplearon nuevos materiales, tales como: acero, placas de yeso cartón, muro cortina con vidrios de colores y revestimientos de madera, que contemplan aproximadamente el 38,4\% del total del edificio.

Los motivos anteriormente expuestos, hacen de este reciclaje un caso pertinente de ser analizado, en términos de vida útil y sostenibilidad de la intervención.

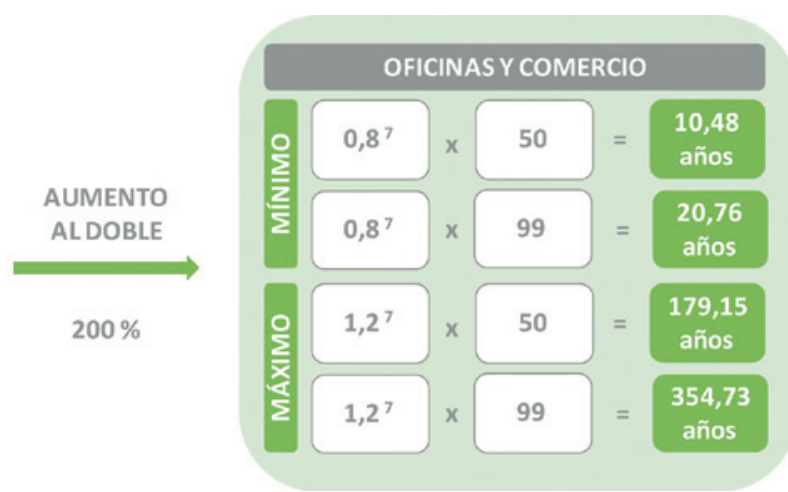

\section{CHV MACHASA: CÁLCULO DE VIDA ÚTIL ESTIMADA (VUE) Y ANÁLISIS DE RESULTADOS}

Las fichas de cálculo A (VUE del uso original: industrias) y B (VUE del uso reciclado: oficinas), se fusionaron, con el fin de demostrar la equivalencia de los criterios empleados en la valorización de cada uno de los 7 factores de durabilidad, obteniendo una clara lectura de estos (Ficha A+B).

En función del cálculo realizado, se establece lo siguiente: 
A5. Zonificación de la Industria Original (izquierda) y Oficinas CHV Machasa (derecha). Fuente: Elaboración propia, a partir de la revisión planimétrica.

A6. Ficha $A+B$ (Comparativa): Cálculo de VUE Caso Estudio. Fuente: Elaboración propia del autor para el análisis de resultados.

- Para el caso original (Ex industria Yarur S.A.): VUD de 25 a 49 años por cinco veces el índice 1,2 (alto) y dos veces el índice 1,0 (medio); expresado como VUD $\times 1,2^{5} \times 1,0^{2}$. De este cálculo se obtuvo como resultado un rango de 62 a 86 años proyectados.

- Para el caso reciclado (CHV Machasa): VUD de 50 a 99 años por seis veces el índice 1,2 (alto) y una vez el índice 0,8 (bajo); expresado como VUD $\times 1,2^{5} \times 0,8^{1}$. De este cálculo se obtuvo como resultado un rango de 119 a 168 años proyectados.

De lo anterior se concluyó que el rango mínimo de VUE fue superado, considerando que la construcción de este tuvo lugar en el año 1935 y la intervención fue realizada en el año 201 1, 76 años después (Figura A6).
(13)

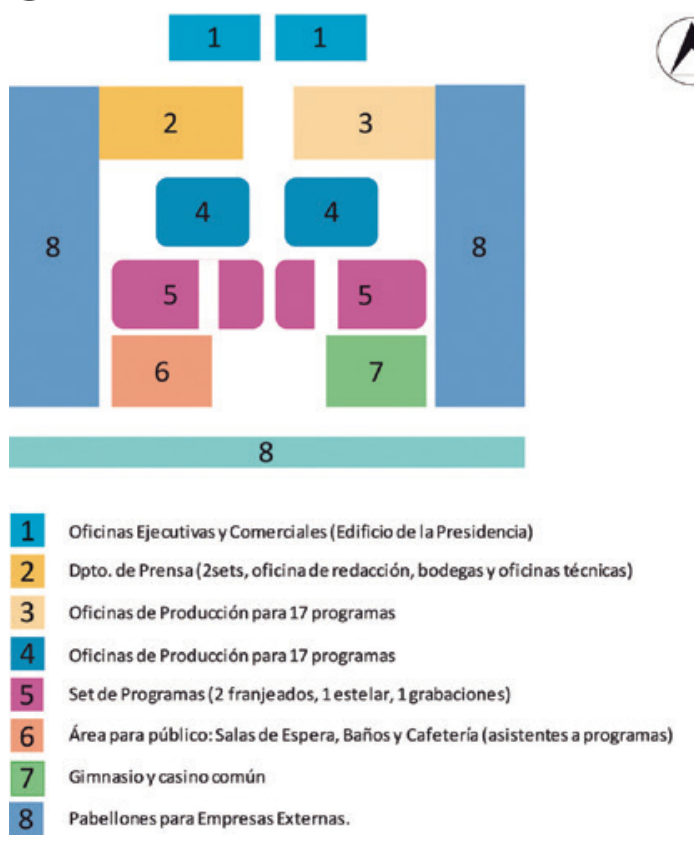

(

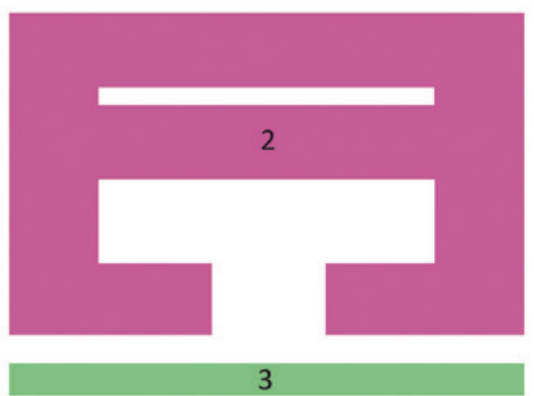

1 Oficinas Ejecutivas y Comerciales (Edificio de la Presidencia)

2 Placa Industrial (Áreade Producciónen Telares)

3 Oficinasy Espacios de Recreación destinados a Trabajadores

\section{(46)}
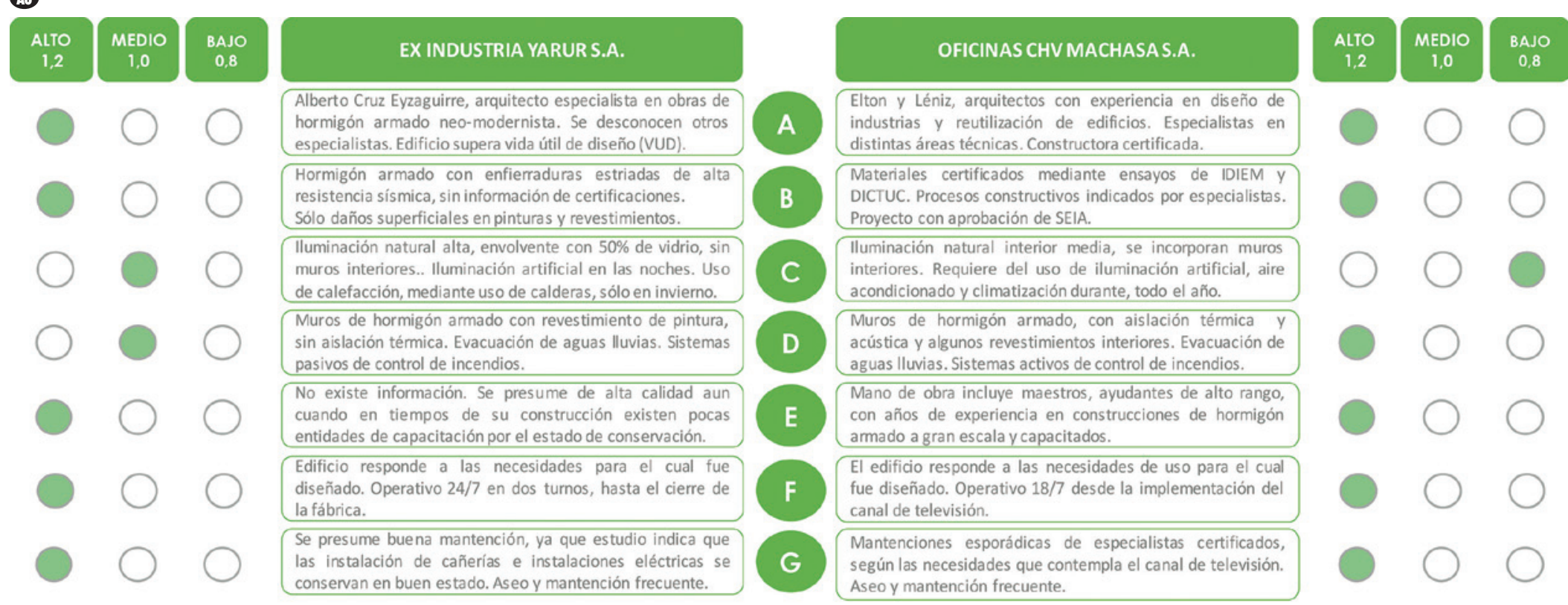

VIDA ÚTIL ESTIMADA

INDUSTRIA

62,21 AÑOS

a

VALORES SEGÚN EL MÉTODO DE FACTORES

VUD
$25 \times 1,2^{5} \times 1,0^{2}$
$49 \times 1,2^{5} \times 1,0^{2}$

VALORES SEGÚN EL MÉTODO DE FACTORES

VUD
$50 \times 1,2^{6} \times \square$
$99 \times 1,2^{6} \times \square \times 0,0,8^{1}$
$\square$

VIDA ÚTIL ESTIMADA

OFICINAS

119,43 AÑOS

a

168,43 AÑOS 
Tras efectuar el cálculo comparativo, se determinó que el reciclaje incrementó entre el $92 \%$ y el $95 \%$ la vida útil del inmueble, en 57-82 años, prolongándose aproximadamente al doble. Si se deseara extender aún más la VUE, se deberían efectuar transformaciones en el diseño haciendo énfasis en el acondicionamiento ambiental interior del edificio, empleando estrategias de iluminación y ventilación pasivas; ya que esto, además, conllevaría a una mejor eficiencia energética del edificio, al incidir directamente en el consumo de electricidad y de los artefactos de climatización (FIgURA A7).

\section{CONCLUSIONES}

Con la estimación de VUE del caso estudio se comprobó que es posible prolongarla al reutilizar la infraestructura con otros fines y es relevante considerar los aspectos constructivos y de gestión, al momento de realizar estas intervenciones que están estrechamente ligados a los nuevos usos, calidad de la intervención, experiencia de los profesionales y medidas de control de variables de deterioro.

Se identificó el incremento de VUE al doble, cuando las industrias en desuso son adaptadas a los nuevos usos de: comercio, oficinas, uso público y edificios patrimoniales, debido a la calidad de sus construcciones y a las constantes mantenciones que se podrían desarrollar en estas tipologías.

Para ello, es importante que el proceso de construcción se realice bajo lineamientos de calidad, considerando la experiencia de los arquitectos, constructores y especialistas involucrados, así como también la mano de obra que debe estar capacitada, para desarrollar todos los procesos constructivos con las técnicas constructivas correspondientes a cada tipo de material. Cabe señalar la importancia que cobran las certificaciones, nacionales o internacionales, en relación con los profesionales y materiales, ya que indican que los edificios han sido construidos con
A7. Gráficas de Factores (arriba); Incremento de Vida Útil (abajo). Fuente: Elaboración propia de la autora, a partir de los resultados obtenidos.
$(47$

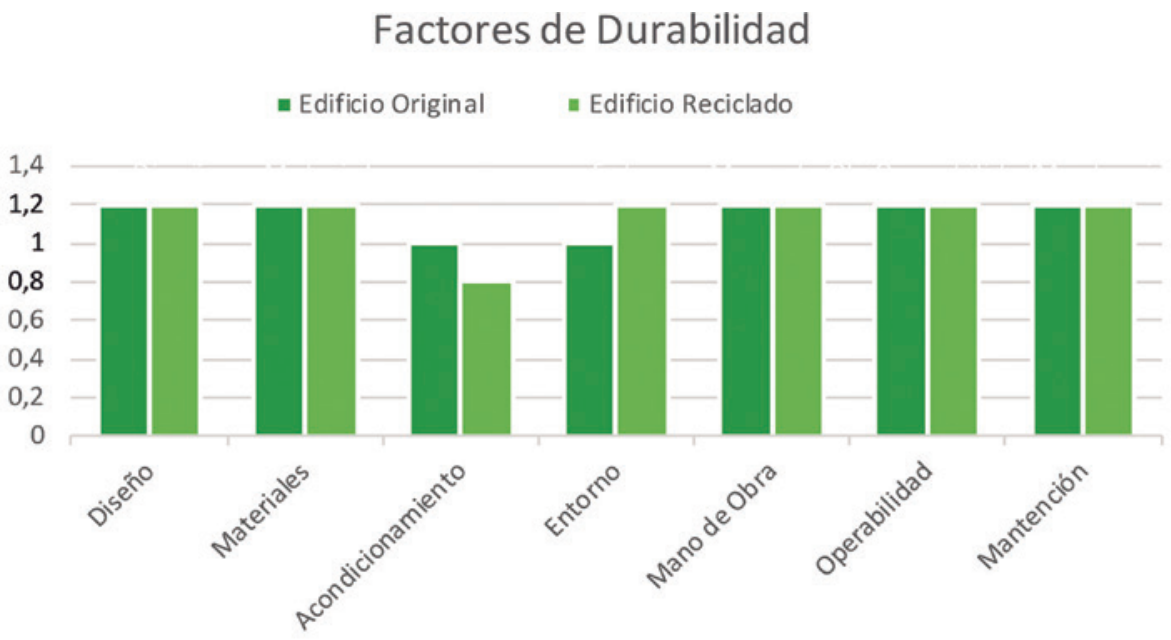

Comparación vida útil estimada

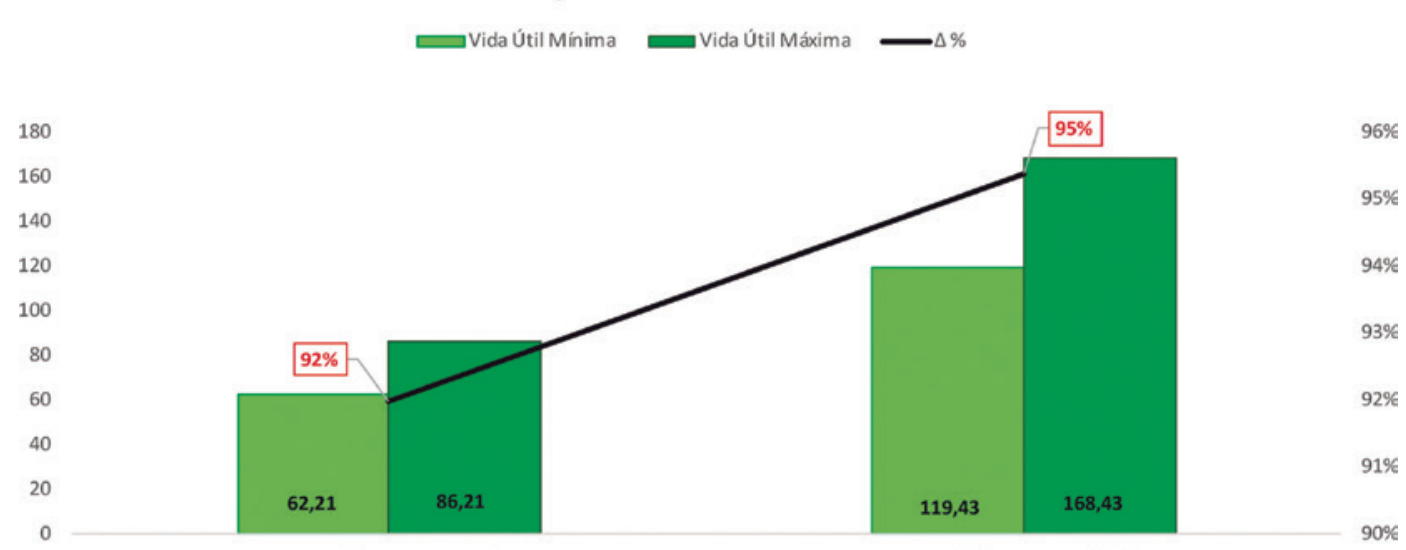

Edificio Original

Edificio Reciclado técnicas de alto nivel constructivo y bajo impacto medioambiental.

Al considerar los factores de durabilidad del edificio se concluye que las intervenciones de los edificios deben poseer un alto acondicionamiento y calidad ambiental interior, haciendo énfasis en las medidas pasivas de ventilación y aprovechamiento de luz natural, con el fin disminuir el consumo de energía operacional y tener mayor durabilidad de las instalaciones de climatización e iluminación. Además, se debe considerar la influencia del entorno y la exposición a distintos cambios climáticos del lugar de emplazamiento y realizar un buen diseño arquitectónico y constructivo, en términos de aislación térmica, filtraciones y sistemas de seguridad. Sin embargo, no es suficiente con la calidad del diseño y construcción, también es necesario que el destino o programa del edificio promueva una alta operatividad, además de una buena mantención e inspecciones postocupación de especialistas con el fin de prolongar o extender la vida útil del inmueble. 
La metodología de cálculo de VUE permite introducir un instrumento sistematizado para la evaluación del estado de las industrias en desuso, según la Norma ISO 15686, para ser utilizado en la etapa de diseño de las intervenciones de estos edificios, tras identificar los factores de durabilidad a potenciar y jerarquizar en la toma de decisiones del proyecto.

En futuras investigaciones será interesante introducir la variable de costo de la obra, indagando en aspectos de sostenibilidad económica y manejo de presupuesto, relacionándolos con los factores de durabilidad del proyecto.

\section{REFERENCIAS BIBLIOGRÁFICAS}

BRE. (2007). Methodology for environmental profiles of construction products, Draft August 2007, BRE, UK.

Canadian Standards Association [CSA]. (2001). Q645-95 (R2001); Life Cycle Costing, pp. 34-52, CSA, Canadá

Canadian Standards Association [CSA]. (2001). S47895 (R2001), Guideline on Durability in Buildings, pp. 9-17, CSA, Canadá.

International Standards Organization (2017). ISO 15686:2000, Buildings and constructed assetsService Life Planning, Part 1-5.

Lillo, M. (2010) Reciclaje de Infraestructuras

Obsoletas. Instituto Universitario de Restauración del Patrimonio de la Universidad Politécnica de Valencia, España.
Hernández, S. (2011). Integration of service life in the process of management and design of buildings. Management Research and Practice. Volumen 2. Marteinsson, B. (2005). Service life estimation in the design of buildings; a development of the factor method. Doctoral Thesis, Research School, Centre for Built Environment, University of Gävle, Suecia.

Hernández, S. \& Ocaña, J. \& Mejía, M. (2015) Application of ISO 15686 to estimate service life of a dome built with adobe technology in the city of Toluca. Universidad Autónoma del Estado, México.

Ortega, L. \& Serrano, B. \& Fran, J. (2015) Proposed method of estimating the service life of building envelopes. Instituto Valenciano de la Edificación de Valencia, España.

\section{EL CEMENTERIO FLOTANTE**}

ARQ. FABIÁN LEIVA CORTÉS · Santiago, Chile ·fabian.leiva@ug.uchile.cl

Fecha de recepción: 28 de septiembre de 2018 . Fecha de aceptación: 28 de noviembre de 2018

"Cada hombre está eternamente ligado a su tierra, no importa la riqueza de la tierra contigua, nadie puede mudarse de tierra, ya que está poseído por ella" (Muntañola, 1998).

Desde el comienzo de la conquista en América, el atropello a las costumbres de las comunidades indígenas que habitan el territorio ha sido reiterado e incluso traspasado entre generaciones, pero si bien hoy esto ha mermado mediante programas que intentan poner en valor lo autóctono, aún quedan conflictos sin resolver. Uno de los más complejos dentro del territorio chileno ha sido la ocupación de tierras ancestrales y sagradas para el pueblo Mapuche, que generalmente se piensa comenzó durante el proceso de colonización, pero la responsabilidad del conflicto actual está lejos de ese origen. No fue sino hasta el siglo XIX que el actual problema se desató con la mal llamada pacificación de la Araucanía, una medida que utilizó el Estado para consolidar su soberanía en una zona considerada rebelde y que dividía al país en dos. Al final del proceso, las comunidades mapuches solamente quedaron en posesión de 500 mil hectáreas, una porción ínfima comparado con los 10 millones que antes poseían. Además, unos 33.000 indígenas quedaron sin tierras o no fueron radicados.
No cuesta entender qué significó esto para un pueblo autodenominado gente de la tierra.

Hoy la lucha continúa, y desde el Estado se siguen aprobando iniciativas que van en contra de las reivindicaciones territoriales que pide este pueblo. Un ejemplo de esto es lo que está sucediendo en la región del Biobío con la construcción de embalses, los que pese a provocar descontento en las comunidades pehuenches de la zona, o la emisión de diversos informes que hablan del daño medioambiental irreparable e incluso problemas socioculturales, siguen avanzando a paso firme gracias a un malentendido progreso.

\footnotetext{
** Realizado en base a los resultados del proyecto de título "El cementerio flotante: arquitectura memorial en la región del Biobío", realizado por el autor en la Facultad de Arquitectura
} y Urbanismo de la Universidad de Chile para obtener el título profesional de Arquitecto (Profesor guía: Albert Tidy Venegas). 
B1. Lugar del desastre, Embalse Ralco, Región del Biobío, Chile. Fuente: https:// coalicionarv.wordpress.com
B2. Estrategia de diseño: la reparación del vínculo perdido. Fuente: elaboración propia.
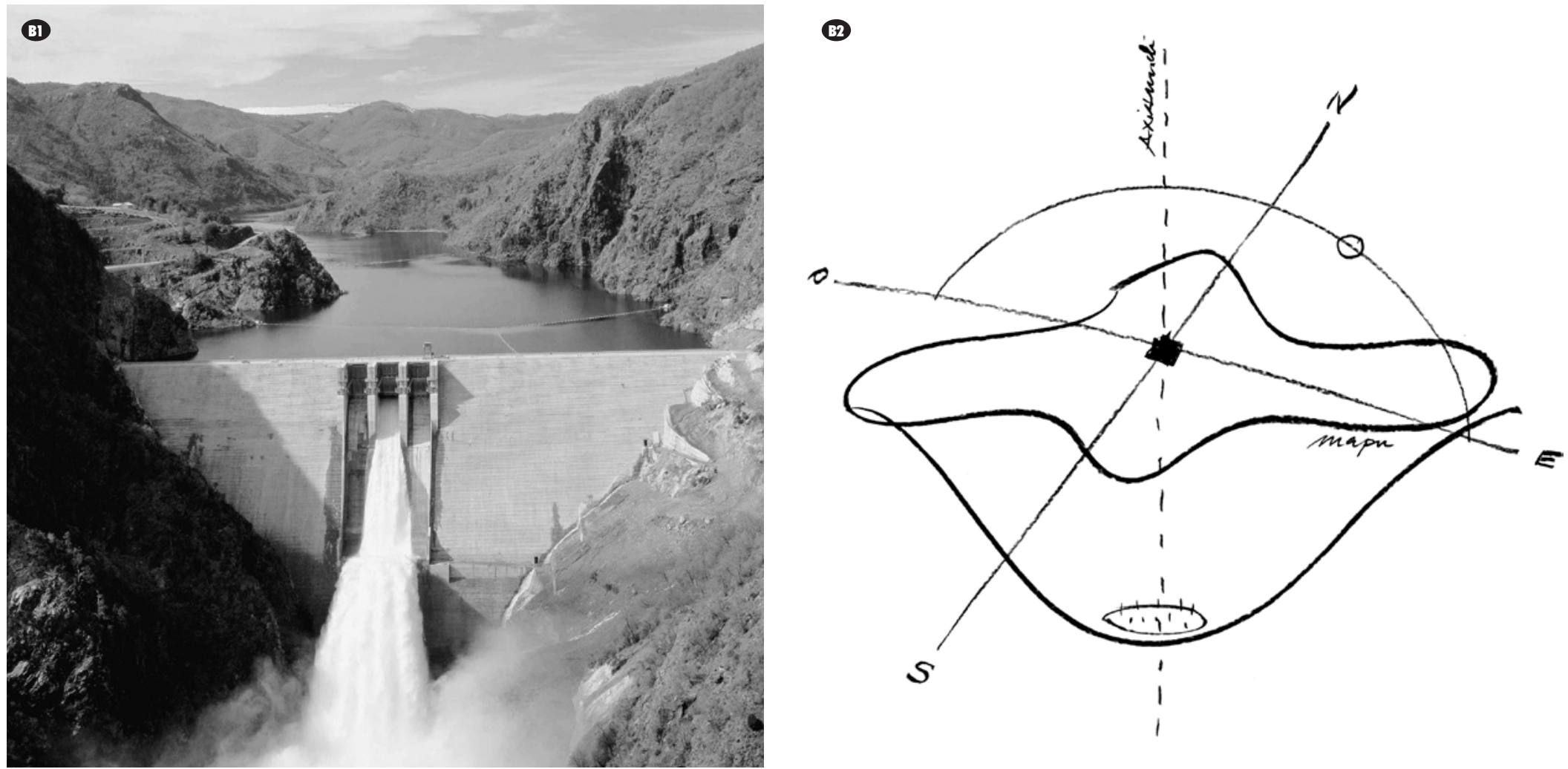

Es en este contexto cuando durante el año 2004 se inunda inesperadamente el Embalse Ralco (Figura B 1) en la Región del Biobío, sur de Chile, sepultando bajo sus aguas el cementerio de la comunidad pehuenche del lugar. Luego de años intentando recuperar los cuerpos, investigaciones demostraron la inviabilidad del rescate, dejando a los pobladores de brazos cruzados frente a millones de metros cúbicos de agua que los separan de sus ancestros.

Hoy el contacto con el cementerio inundado está relegado a las orillas del embalse, y solo para el pequeño grupo de familias que aún tienen en la memoria la existencia del cementerio

Quepuca. Allí se ha puesto una modesta cruz de madera que relata lo ocurrido, y donde cada tanto la comunidad se reúne y realiza rogativas para recordar a los familiares que quedaron sepultados bajo el agua; según ellos los espíritus se los piden.
De esta tragedia nace la idea de crear un espacio para la memoria de un lugar sagrado vulnerado e imposible de recuperar, que acerque la comunidad a sus muertos y sea una eterna condena al hecho. La cosmovisión pehuenche considera nuestro mundo como parte de una serie de plataformas cuadradas superpuestas conectadas verticalmente por un eje, lo que permite entender que la comunicación entre los distintos planos se establece mediante una relación vertical (Figura B2). En base a estas creencias, se proyecta un punto de encuentro entre vivos y muertos, posicionando sobre las aguas del embalse un elemento mono-material de hormigón que establezca una relación vertical con el cementerio inundado (FIGURA B3), generando una puerta o umbral al mundo de los muertos a través de un volumen simple y puro, carente de cualquier simbolismo, pero cargado de contenido en su espacialidad (FIgura B4), encargado de perpetuar la tarea de recordar y reunir a la comunidad de forma indefinida.

Para este propósito, se toma como referencia la plataforma cósmica en que habitamos (Mapu), y se genera una sustracción de su centro, marcando allí el punto de conexión entre el eje cósmico y el plano terrenal, la puerta entre vivos y muertos (Figura B5), que señalará también la ubicación exacta del cementerio inundado. Entre el espacio central y el perímetro del volumen se generará un vacío que permitirá la flotabilidad del elemento. En esta área se dará una instancia íntima de recuerdo, donde alrededor de 700 nichos vacíos recordarán el espacio que dejan los muertos sepultados por el agua, dando así la escala al proyecto (Figuras B6 y B7). Aquí se albergará la continuación de un rito fúnebre tradicional, que posibilitará a quienes no pudieron yacer junto a sus ancestros ser repatriados a este lugar mediante el 
B3. Planta de emplazamiento. Fuente: elaboración propia.

B4. Vista general del proyecto: punto de encuentro entre vivos y muertos. Fuente: elaboración propia.

B5. Isométrica explotada. Fuente: elaboración propia.

B6. Planta del primer nivel. Fuente: elaboración propia.
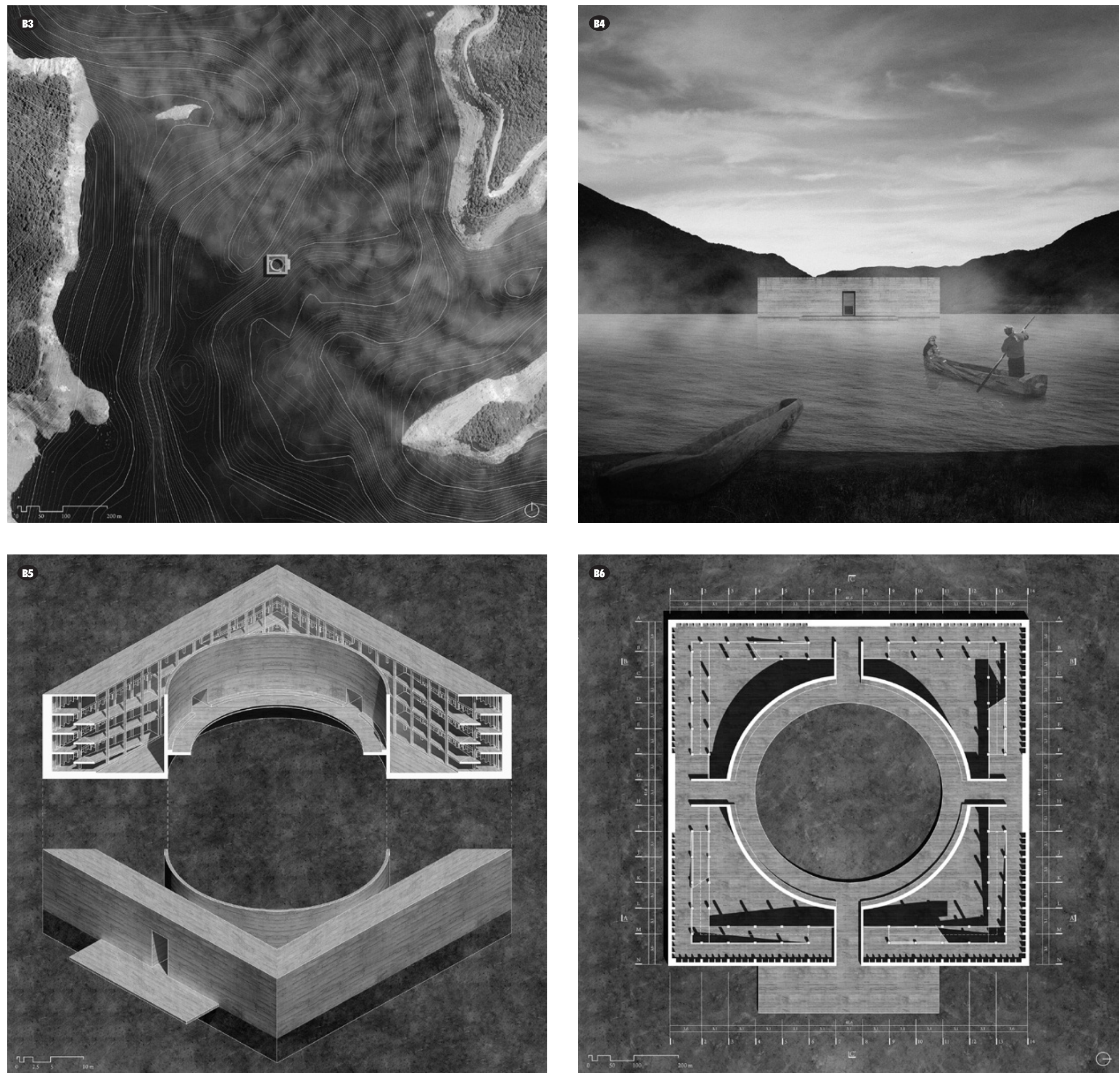
B7. Secciones del proyecto. Fuente: elaboración propia.
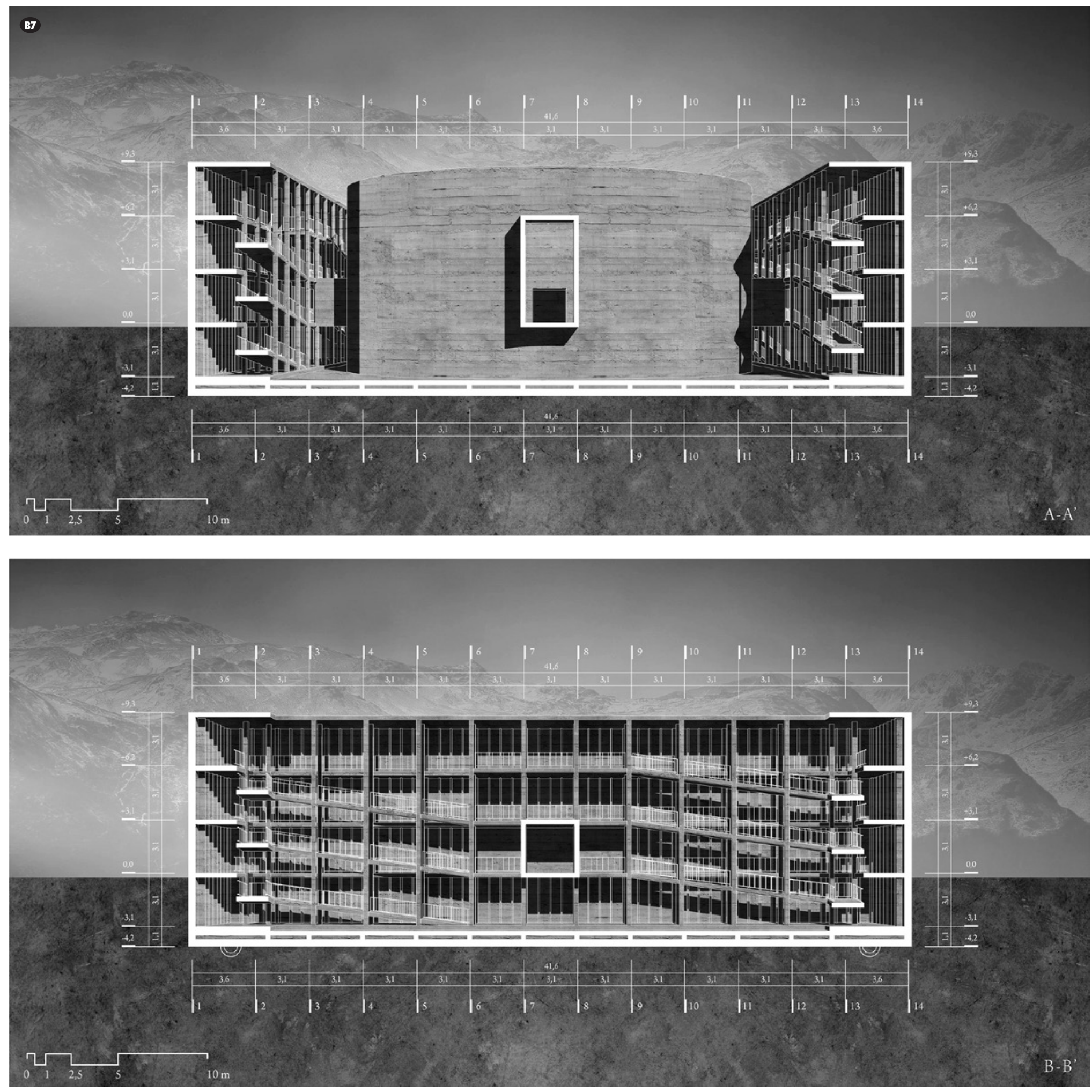
B8. Imágenes del proyecto. Fuente: elaboración propia.

reposicionamiento dentro del memorial de su chemamull, estatua de madera antropomorfa que señala el lugar donde descansa el cuerpo, guardianes del espíritu; llenando así poco a poco los espacios dejados por sus antepasados.

Es de esta manera como se busca sacar a flote una verdad que intento ser olvidada, proyectando una obra cuya complejidad no se encuentra en sus características formales o materiales, sino en la sensibilidad con que se aproxima a resolver un problema que parece irreparable, cuyo usuario tiene además una forma muy particular de entender el cosmos y la comunicación entre sus distintos planos, tarea solo posible de concretar cuando se entiende que la conexión se da principalmente en un plano espiritual (FIGURA B8).

Cuando un lugar es destruido, se destruye también su espíritu, pero si en algo puede ayudar la arquitectura es a entender un problema más allá de lo meramente físico, y generar lugares que no busquen ser un reemplazo de otro, sino la siguiente etapa de lo que allí ocurría.

\section{REFERENCIAS BIBLIOGRÁFICAS}

Muntañola, J. (1998). La arquitectura como lugar. Barcelona: UPC.
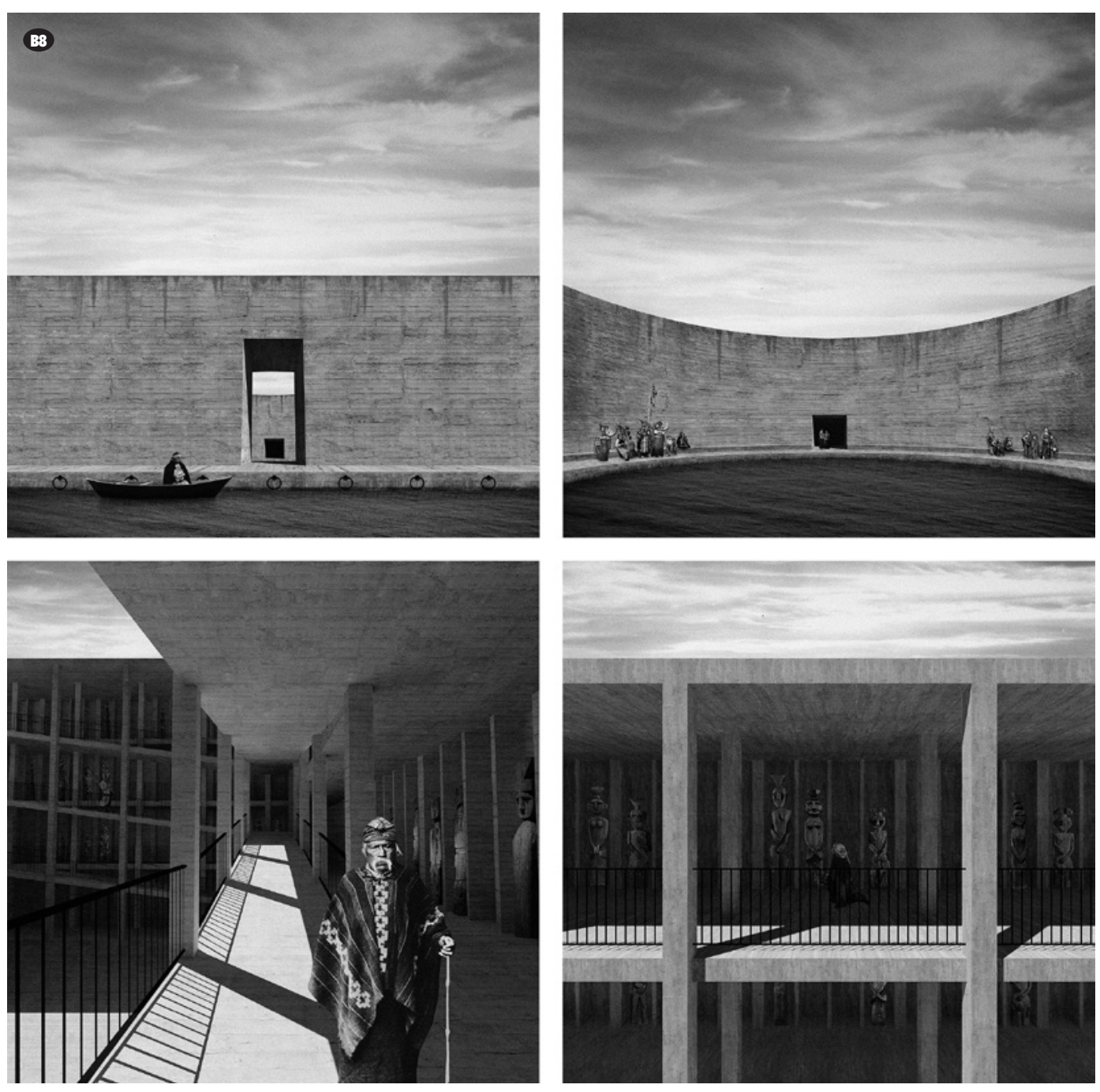